\title{
Exploring left-right symmetry of somites through multiview light sheet microscopy
}

Sundar Naganathan, Marko Popovic, Andrew Oates École Polytechnique Fédérale de Lausanne, Switzerland

\section{Abstract Text}

The body axis of vertebrate embryos is periodically segmented into bilaterally symmetric pairs of somites, which gives rise to the adult musculoskeletal system. The anteroposterior (AP) length of somites and their left-right symmetry is generally thought to be determined in the unsegmented presomitic mesoderm by genetic oscillations of a segmentation clock and downstream molecular prepatterns. While mechanical processes have also been associated with somite morphogenesis, their role in determining AP length and left-right symmetry, if any, is not understood. In general, a quantitative study of bilateral symmetry in somites is lacking owing to the technical difficulty in following 3D somite morphogenesis simultaneously on the left and right sides of embryos.

To address this, we performed multiview light sheet microscopy of zebrafish embryos and followed morphogenesis of the first eight somites on both left and right sides simultaneously. We show that initial somite lengths and positions are imprecise and many somites form left-right asymmetrically. This suggests that left-right somite formation should not be assumed to be a default state, as one typically reads in reviews. Strikingly, within an hour, lengths are adjusted, becoming more symmetric, through changes in somite shape occurring independently on the left and right sides of the embryo. This adjustment is directed by a combination of somite surface tension, external stresses from neighbouring tissues and convergence-extension flows within somites. The ensuing left-right symmetry in somite boundary positions follows as a straightforward consequence of the length adjustments. Thus, the precision and symmetry of a fundamental embryonic morphological pattern is ensured by tissue mechanics. 
